\title{
Limnophila (Scrophulariaceae): Chemical and Pharmaceutical Aspects - An Update
}

\author{
Goutam Brahmachari*
}

Laboratory of Natural Products \& Organic Synthesis, Department of Chemistry, Visva-Bharati (a Central University), Santiniketan-731 235, West Bengal, India

\begin{abstract}
The present resumé covers an up-to-date literature on Limnophila species. The botanical classification, ethnopharmacology, and chemical constituents of Limnophila plants, as well as the biological activities and pharmacological applications of both distinct phytochemicals and medicinally active plant materials (formulations, extracts, etc.) are discussed in detail.
\end{abstract}

Keywords: Biological activities, Botany, Chemical constituents, Ethno-pharmacology, Limnophila species, Pharmacological applications, Taxonomical classification.

\section{INTRODUCTION}

Limnophila (family: Scrophulariaceae) [1-5] is originated from a Latin word that means pond-loving indicating its existence in aquatic environments. It is commonly known as 'Ambulia' (Asian marshweed). It is a perennial herb from Southeast Asia, tropical to subtropical Africa, Australia, and Pacific Islands; also finds adventive distribution in North America. Limnophila plants are widely distributed throughout India, and occupy a significant position in traditional systems of medicine. A number of plant species are in use as folk medicines in the treatment of various ailments. A number of works on chemical and pharmacological aspects of genus Limnophila have already been done. Here an attempt, for the first time as per our record, has been made to compile all these works that are in scattered in literatures. Although some works on the genus have been done, a major portion remains unexplored. This review is designed in such a fashion so that it would surely boost the ongoing research in this direction. That's why an all round and up-to-date resumé covering its botany to ethnobotany, biological and pharmacological studies as well as phytochemicals as reported so far —on this important plant genus has been compiled.

\section{BOTANICAL ASPECTS}

Limnophila $[6,7]$ is an aquatic, or nearly aquatic, perennial herb found as submersed, emergent, and amphibious stem plant. Its natural habitats are rivers, lakes, ponds as well as marshy lands. The submerged stems are smooth and have feathery leaves with $30 \mathrm{~mm}$ long encircling about the stems. These differ from the emergent stems, which are covered with flat shiny hair and have leaves, generally lance-shaped, up to $3 \mathrm{~cm}$ long with toothed margins. Stems may be up to 12

*Address correspondence to this author at the Laboratory of Natural Products \& Organic Synthesis, Department of Chemistry, Visva-Bharati (a Central University), Santiniketan-731 235, West Bengal, India;

Tel: +91-3463-261526; Fax: +91-3463-261526;

E-mails: brahmg2001@yahoo.co.in and brahmg2001@gmail.com feet long. The emergent stems are usually $2-15 \mathrm{~cm}$ above the surface of the water. Single white, pink, purple or blue to lavender flowers, sometimes with conspicuous spots, occasionally occur on the emerged portion of the stem. The flowers are stalkless and borne in the leaf axis, and are axillary and solitary or in axillary or terminal spikes or racemes, sessile or pedicellate. The lower portion (sepals) has five, green, hairy lobes, each $4-5 \mathrm{~mm}$ long. The upper portion is purple and composed of five fused petals forming a tube with two lips - adaxial lip (dorsal) is 2-lobed, while abaxial lip (ventral) is 3-lobed. The lips have distinct purple lines on the undersides. The fruit is capsule containing up to 150 seeds.

Limnophila reproduces through fragmentation of the stem or by seeds. In post-rainy session the fruits of Limnophila are mature, and the mats break loose from the hydrosoil - as the floating-mats move, they spread out the seeds throughout a wider area.

\section{TAXONOMICAL BACKGROUND}

The taxonomical classification $[8,9]$ of Limnophila plants are shown below:

$\begin{array}{ll}\text { Kingdom } & \text { Plantae } \\ \text { Subkingdom } & \text { Tracheobionta } \\ \text { Division } & \text { Magnoliophyta } \\ \text { Class } & \text { Magnoliopsida } \\ \text { Subclass } & \text { Asteridae } \\ \text { Order } & \text { Scrophulariales } \\ \text { Family } & \text { Scrophulariaceae } \\ \text { Genus } & \text { Limnophila } \text { R. Brown }\end{array}$

About 40 species [10] of the genus Limnophila are known; some common species are cited here: 


\begin{tabular}{|c|c|}
\hline L. aromatica (Lamarck) Merrill. & L. laotica Bonati \\
\hline (Syn. L. aromaticoides Yang \& Yen; & L. laxa Bentham \\
\hline L. gratissima Blum) & L. micrantha (Benth.) Bentham \\
\hline L. australis Wannan \& Waterh. & L. parviflora Yamazaki \\
\hline L. balsamea (Benth.) Benth. & L. poilanei Yamazaki \\
\hline (Syn. L. thorelii Bonati) & L. polyantha Kurz ex Hook.f. \\
\hline L. borealis Y. Z. Zhao \& Maf. & (Syn. L. polyantha Yamazaki) \\
\hline L. brownii Wannan & L. repens (Bentham) Bentham \\
\hline L. chinensis (Osbeck) Merill & (Syn. L. conferta Bentham; \\
\hline (Syn. L. chevalieri Bonati; L. hirsuta & L. dubia Bonati; L. sessilis \\
\hline (Heyne ex Benth.) Benth. & (Bentham) Fischer \\
\hline L. connata (Buchanan-Hamilton ex D. & L. rugosa (Roth) Merill \\
\hline Don) & (Syn. L. roxburghii G. Don) \\
\hline Handel-Mazzetti & L. sessiliflora (Vahl) Blume \\
\hline L. erecta Bentham & L. siamensis Yamazaki \\
\hline L. fragrans Seem & L. taoyuanensis Yang \& Yen \\
\hline L. geoffrayi Bonati & L. verticillata Yamazaki \\
\hline L. hayatae Yamazaki & L. villifera Miq. \\
\hline L. heterophylla (Linnaeus) Druce (Syn. & L. X ludoviciana Thieret \\
\hline L. reflexa Bentham) & L. dasyantha Skan \\
\hline L. indica (Linnaeus) Druce (Syn. $L$. & L. glabra (Benj.) Kerr \\
\hline gratioloides R. Brown; L. racemosa & L. hottonoides Druce \\
\hline Bentham; L. aquatica Roxburgh) & L. gigantean \\
\hline
\end{tabular}

\section{TRADITIONAL USES}

Limnophila plants are extensively used in the indigenous system of medicine, and are found to be useful and effective. Traditional uses of only a few of these significant plant species finding useful applications in the treatment of various ailments are mentioned here. The medicinal uses of these plant species are being cited on the basis of extensive literature survey.

(i) L. aromatica (Syn. L. gratissima): The plant is used as a spinach, eaten raw or steamed. It is sour, slight bitter refrigerant emollient antiseptic, galactagogue, aperient, appetizer, digestive, carminative, anthelmintic, antiinflammatory, diuretic and febrifuge. It is useful in vitiated conditions of pitta, foul ulcers, agalactia, galactic impurities, anorexia, dyspepsia, helminthiasis, constipation, inflammations and strangury. The juice of the plant is used as a cooling medicine in fever and pharyngitis. It is given to nursing women, when the milk is sour. The plant emits turpentine-like odour and yields an essential oil [11-13].

(ii) L. rugosa (Syn. L. roxburghii): The plant shows numerous medicinal applications in the traditional system. The juice of the plant is rubbed over the body in pestilent fever. It is applied on elephantiasis with coconut oil. It is administered in diarrhoea, dysentery and dyspepsia. It is used as carminative and tonic. The essential oil is used as flavouring agent of food and perfuming of hair oils. The essential oil of this plant also exhibits significant anti-bacterial and anti-fungal activities. The plant had been accepted for "Sugandhabala" as it responded to Ayurvedic description of the drug. Infusion of leaves is used as diuretic and stomachic in the Philippine Islands and in India [12, 14, 15].

(iii) L. indica (Syn. L. gratioloides; L. racemosa; L. aquatica): The plant has a refreshing and agreeable odour resembling to camphor or oil of lemon. L. indica is considered to be carminative and antiseptic. A liniment prepared from the plant is used in elephantiasis. The juice of the plant is rubbed over the body in pestilent fevers. It is given internally in dysentery combined with ginger, cumin and other aromatics [12-14].

(iv) L. conferta: The plant has been employed to treat various types of skin diseases and conditions of inflammation in the indigenous system of medicine $[14,16]$.

\section{CHEMICAL CONSTITUENTS OF LIMNOPHILA}

The phytochemical investigation of the genus Limnophila, as carried out so far, has afforded a good number of compounds, previously known from other natural sources or isolated as new phytochemicals. These compounds are of varying structural skeletons and are classified into flavonoids (1-23; Fig. 1) (Table 1), terpenoids (24-60; Fig. 2) (Table 2), and miscellaneous (61-87; Fig. 3) (Table 3).

\section{BIOLOGICAL/PHARMACOLOGICAL ACTIVITIES OF CRUDE PLANT MATERIALS AS WELL AS OF CHEMICAL CONSTITUENTS}

A good number of biological/pharmacological works with different parts of Limnophila plants as crude extracts and also of pure chemical constituents isolated from these plant species have been reported so far. This section is an attempt to sum up all these findings.

\section{Antimicrobial Activity}

Limnophila plants are reported to exhibit significant antimicrobial activity. L. racemosa and $L$. indica extracts were found to inhibit the growth of Xanthomonas campestris and $X$. malvacearum in vitro [50]. Mishra et al. [51] also studied the antimicrobial activity of the same plant extracts against a number of bacterial species and obtained a convincing result (Table 4) on the basis of which the workers pointed out that both the extracts of $L$. racemosa and $L$. indica bear certain antimicrobial components.

Antibacterial efficacy of the essential oil of L. conferta was also established by Reddy et al. [33] against the Grampositive bacteria, Staphylococcus aureus and Bacillus subtilis, and two Gram-negative bacteria, Escherichia coli and Pseudomonas aeruginosa. The significant antimicrobial activity of the essential oil of $L$. conferta, comparable with that of chloramphenicol used as standard and ethylene glycol as control solvent justifies the use of this plant in the indigenous system of medicine in controlling some infections. The oil was not found to be toxic at a dose level of $1.6 \mathrm{~mL} / \mathrm{Kg}$ orally.

Reddy et al. [33] reported that the essential oil of $L$. gratissima (Syn. L. aromatica) shows a good antimicrobial activity (Table 5) of the same order of that of the reference standards, streptomycin and chloramphenicol. Recently, antimicrobial efficacy of the plant extracts against number bacterial strains was found to be promising [52-54].

Further, Kapil et al. [55] reported that Limnophila rugosa essential oil and its constituents also show potent anti-bacterial activity against Bacillus subtilis and 
<smiles>COc1ccc(-c2cc(=O)c3c(O)c(OC)c(OC)cc3oc2=O)cc1</smiles>

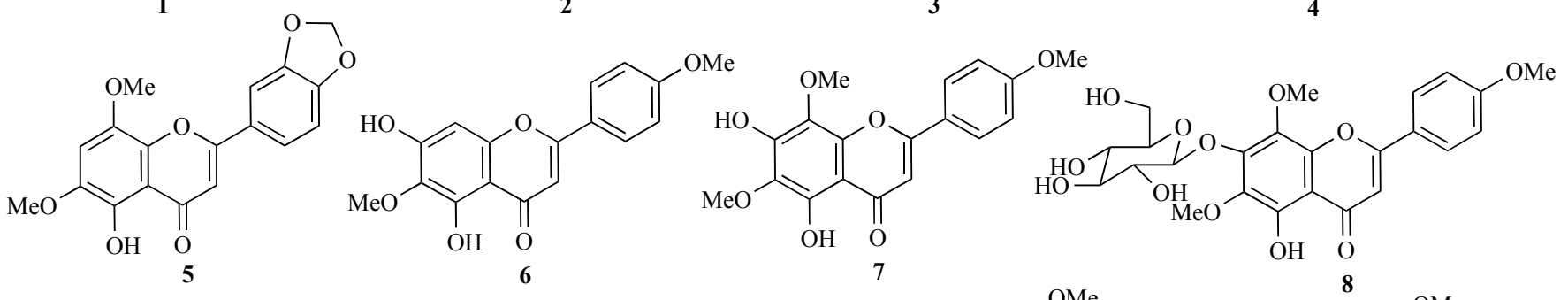<smiles>C#Cc1cc(-c2cc(OC)cc(OC)c2)cc(=O)c2c(O)c(O)cc(O)c12</smiles><smiles>COc1ccc(-c2cc(=O)c3c(O)cc(OC)c(OC)c3o2)c(O)c1</smiles><smiles>COc1ccc(-c2cc(=O)c3c(O)c(OC)c(O)c(O)c3o2)cc1</smiles><smiles>COc1cc(OC)c2c(c1)O[C@@H](c1ccc(OC)c(OC)c1)CC2=O</smiles>

Fig. (1). Structures of flavonoids from Limnophila.

Salmonella typhi. Chloroform extract of the aerial parts of $L$. geoffrayi also found to possess antimycobacterial activities [24]. The essential oil of L. rugosa is reported to exhibit antifungal activity [13]. The essential oil of $L$. conferta is also a useful antifungal antidote. The antifungal activity of the oil at 1: 50 dilution in ethylene glycol was found to be of the same order as that of griseofulvin in chloroform used as standard $(100 \mu \mathrm{g} / 0.1 \mathrm{ml})$. In case of the dermatophytes viz. Trichophyton mentagrophytes and Microsporum gypseum the oil at a concentration of $100 \mu \mathrm{g} / \mathrm{mL}$ inhibited the growth of both the fungi, however less effective than the standard, miconazole $(10 \mu \mathrm{g} / \mathrm{mL})$ [33].
A promising antifungal efficacy of L. gratissima (essential oil) was reported by Rao et al. [56]. At a dose of $0.1 \mathrm{~mL}$, the essential oil of the plant showed inhibition zones of 20, $28 \& 25 \mathrm{~mm}$ (diameter) respectively against Aspergilus niger, Rhizopus oryzae and Candida albicans while the reference standard, griseofulvin exhibited the respective inhibition zones of 18,24 and $14 \mathrm{~mm}$ at a dose of $100 \mathrm{mg}$ in $\mathrm{CHCl}_{3}$. It appeared that the oil of L. gratissima is mostly active against Rhizopus oryzae, and the efficacy is greater than grisofulvin; thus the above findings are in support with the traditional uses of the plant oil as antiseptic [56]. 
Table 1. Flavonoid Constituents of Limnophila.

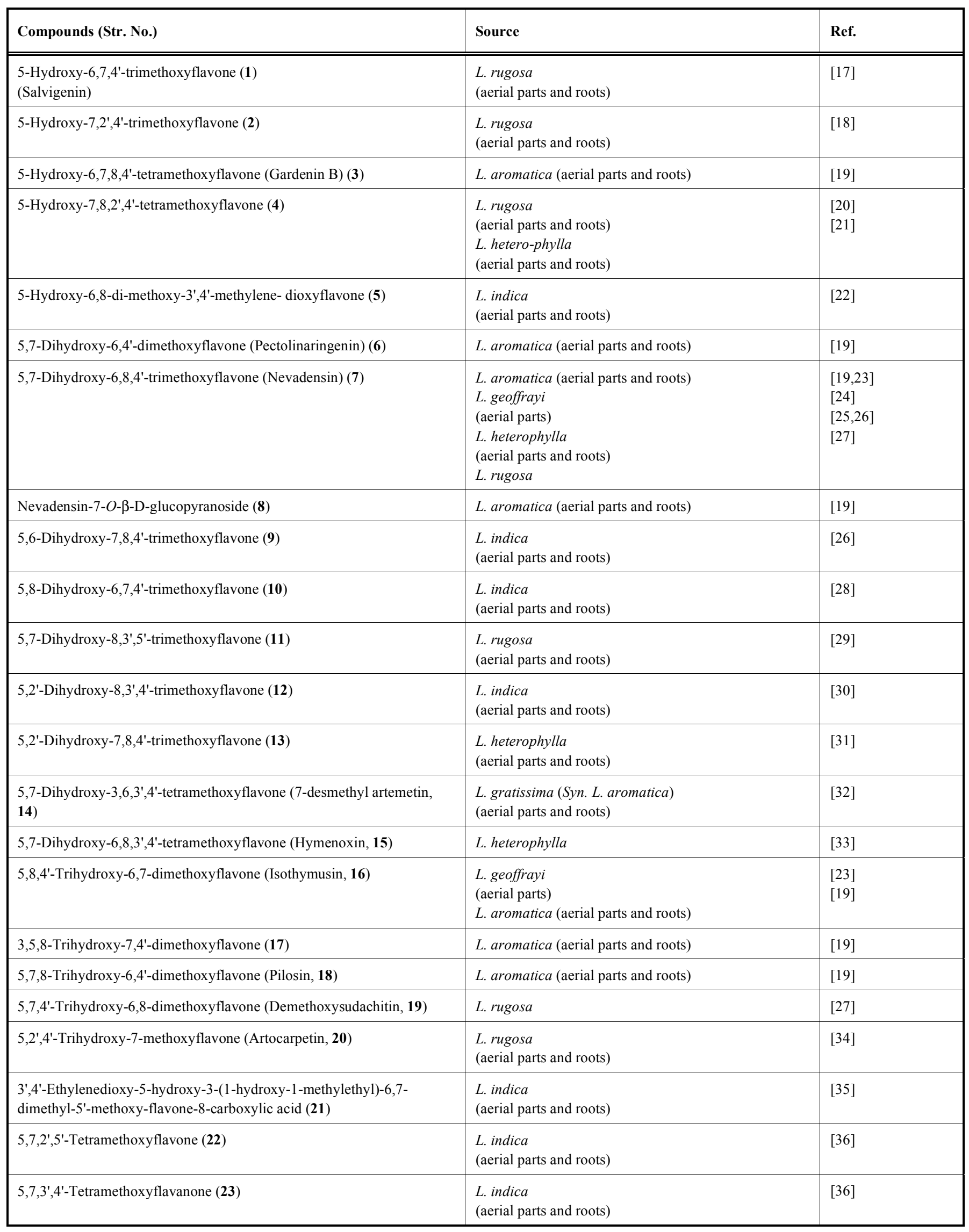




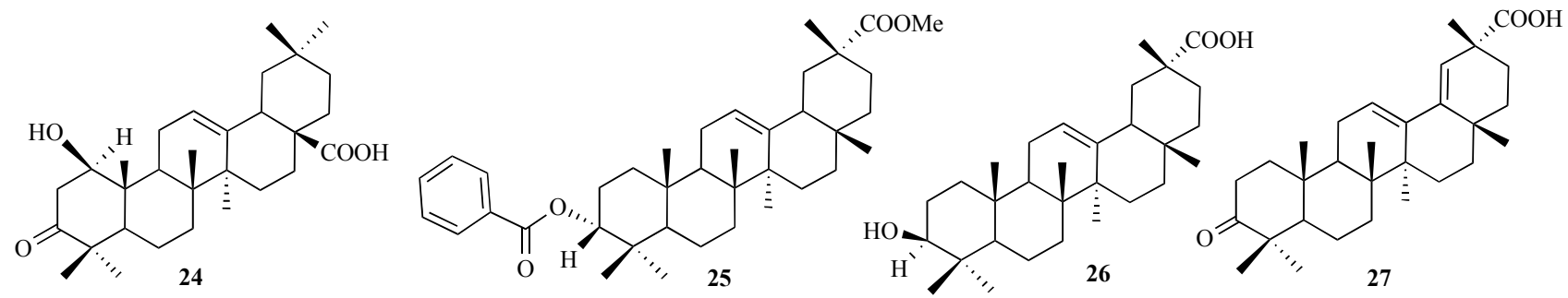

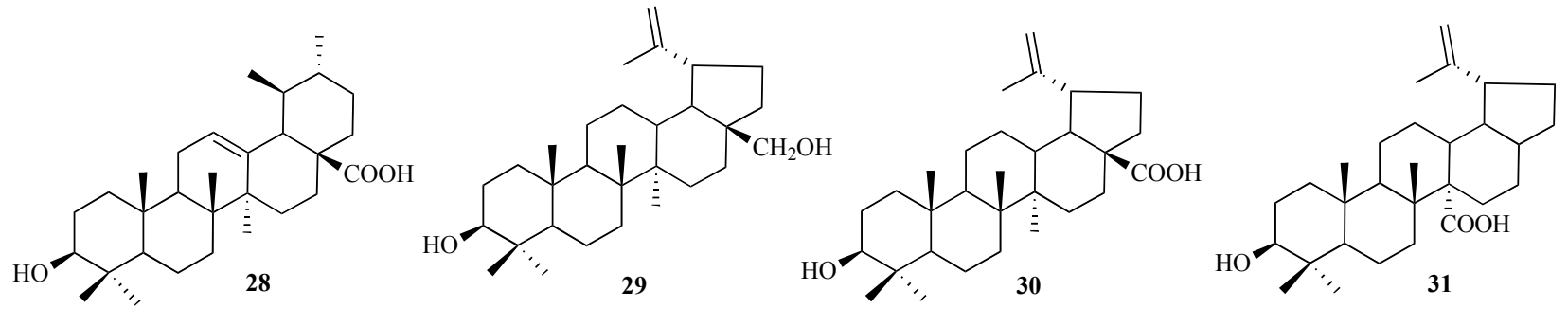<smiles>C=C[C@](C)(O)CC/C=C(/C)C/C=C(/C)C/C=C\C(C)(C)C</smiles><smiles>C=C1CC/C=C(/C)CCC2CC1CC2(C)C</smiles><smiles>CC1=C[C@H]2C(C)=CC[C@H](C(C)C)[C@H]2CC1</smiles><smiles>CC1=CCC2CC1CC21CC1</smiles><smiles>C=C1CC2CCC1C(=C)C2(C)C(C)(C)C1CCC2(C(C)C)CCC1C2</smiles><smiles>CC1=C[C@@H]2C(CC1)C2(C)C</smiles>

41
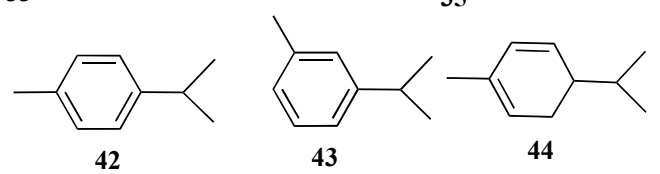

43<smiles>CC1=CCC(O)(C(C)C)CC1</smiles>

49

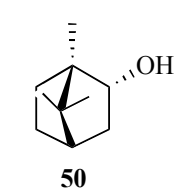

48

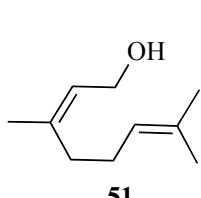

51

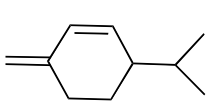

45<smiles>C=C/C(C)=C/CC=C(C)C</smiles>

46<smiles>C=CC(=C)CCC=C(C)CCC=C(C)C</smiles>

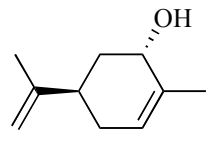

55

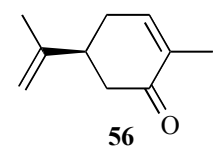

56<smiles>CC(C)=CCC/C(C)=C/CO</smiles>

57<smiles>CC1CC(=O)C(C(C)C)C1</smiles><smiles>CC1=CCC[C@]2(C)CCC(C(C)(C)O)C[C@H]12</smiles><smiles>C=C(C)[C@H]1CC=C(C=O)CC1</smiles><smiles>C=C(C)[C@@H]1CC[C@@]2(C)OC2C1</smiles>

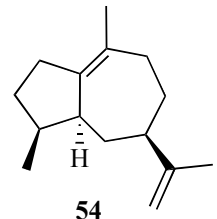

54<smiles>C=C/C(C)=C\CC=C(C)C</smiles>

Fig. (2). Structures of terpenoids from Limnophila.

Methanolic extract of the whole plants of $L$. indica was found to possess potent antidiarrheal and anti-dysentery efficacy with mild antacid activity. Subhadra et al. [57] performed both in vitro and in vivo studies to evaluate the activities. In vitro antibacterial studies with the methanolic extract revealed that at $100 \mathrm{mg} / \mathrm{ml}$ it showed more potent activity than gentamycin at $1 \mathrm{mg} / \mathrm{ml}$ against $S$. aureus and $E$. coli, while exhibiting similar antibacterial potency as that of gentamycin against the bacterial strains $P$. aeruginosa and $B$. substilis. The study further unearthed an excellent activity of L. indica against three Shigella species such as S. flexineri, $S$. dysentery and S. boydii. It is worthy to mention that the plant extract was found to have greater efficacy against $S$. flexineri than the standard drug ceftazidime. The zones of inhibition (in $\mathrm{mm}$ scale) for the drug (at $1 \mathrm{mg} / \mathrm{ml}$ ) and the extract (at $100 \mathrm{mg} / \mathrm{ml}$ ) were measured as $15.0 \pm 0.0 \& 18.33 \pm 0.58$ against $S$. flexineri, $28.0 \pm 1.0 \& 18.67 \pm 0.58$ against $S$. dysentery and $20.0 \pm 0.0 \& 19.0 \pm 0.0$ against $S$. boydii, respectively [57]. Compared with the normal control group, both the metahnolic extract and ceftazidime were found to be more active against the Shigella species $(\mathrm{P}<0.01)$. The minimum inhibitory concentrations (MICs) for the methanolic plant extract to inhibit the growth of S. flexineri, S. dysentery and $S$. boydii were measured as 230, 233 and $235 \mu \mathrm{g} / \mathrm{ml}$, respectively. The authors further performed in vivo experiments to disclose antidiarrheal activity of the $L$. indica plant extract; the methanolic plant extract at a lower dose of 100 $\mathrm{mg} / \mathrm{kg}$ showed more potent antidiarrheal activity than that of loperamide (at $3 \mathrm{mg} / \mathrm{kg}$ ), a standard drug having several side-effects. Hence, this plant can be a potent substitute for the synthetic antidiarrheal and anti-dysentery drugs [57]. 
Table 2. Terpenoid Constituents of Limnophila.

\begin{tabular}{|c|c|c|}
\hline $\begin{array}{l}\text { Compounds } \\
\text { (Str. No.) }\end{array}$ & Source & Ref. \\
\hline $1 \beta$-Hydroxy-3-keto-olean-12-en-28-oic acid (24) & $\begin{array}{l}\text { L. rugosa } \\
\text { (aerial parts and roots) }\end{array}$ & {$[37]$} \\
\hline Methyl-olean-12-ene-3 $\alpha$-benzoyloxy-29-carboxylate (25) & $\begin{array}{l}\text { L. heterophylla } \\
\text { (aerial parts and roots) }\end{array}$ & {$[38]$} \\
\hline 3-Oxo-olean-12(13),18(19)-dien-29 $\alpha$-carboxylic acid (27) & $\begin{array}{l}\text { L. indica } \\
\text { (aerial parts and roots) }\end{array}$ & {$[40]$} \\
\hline Ursolic acid (28) & $\begin{array}{l}\text { L. heterophylla } \\
\text { (aerial parts and roots) } \\
\text { L. rugosa } \\
\text { (aerial parts and roots) }\end{array}$ & $\begin{array}{l}{[21]} \\
{[41]}\end{array}$ \\
\hline Betulinic acid (30) & L. rugosa & [42] \\
\hline 3ß-Hydroxy-lup-20(29)-en-27-oic acid (31) & L. rugosa & {$[27]$} \\
\hline$R$-(+)-Limonene (32) & $\begin{array}{l}\text { L. heterophylla (Essential oil) } \\
\text { L. geoffrayi (Essential oil) }\end{array}$ & $\begin{array}{l}{[43]} \\
{[44]}\end{array}$ \\
\hline$R$-Linalool (33) & $\begin{array}{l}\text { L. rugosa }(\text { Essential oil) } \\
\text { L. aromatica }(\text { Essential oil) } \\
\text { L. geoffrayi }(\text { Essential oil) }\end{array}$ & $\begin{array}{l}{[43]} \\
{[45-47]} \\
{[44]}\end{array}$ \\
\hline$\alpha$-Humulene (34) & L. rugosa (Essential oil) & [39] \\
\hline$\beta$-Caryophyllene (35) & $\begin{array}{l}\text { L. rugosa }(\text { Essential oil) } \\
\text { L. aromatica }(\text { Essential oil) } \\
\text { L. geoffrayi (Essential oil) }\end{array}$ & $\begin{array}{l}{[39]} \\
{[45-47]} \\
{[44]}\end{array}$ \\
\hline$\beta$-Pinene (38) & $\begin{array}{l}\text { L. aromatica (Essential oil) } \\
\text { L. geoffrayi (Essential oil) }\end{array}$ & $\begin{array}{l}{[47]} \\
{[44]}\end{array}$ \\
\hline Camphene (39) & L. aromatica (Essential oil) & {$[45,46]$} \\
\hline$(+)$-Sabinene (40) & $\begin{array}{l}\text { L. aromatica }(\text { Essential oil) } \\
\text { L. geoffrayi (Essential oil) }\end{array}$ & $\begin{array}{l}{[45,46]} \\
{[44]}\end{array}$ \\
\hline 2-Carene (41) & L. aromatica (Essential oil) & {$[44-46]$} \\
\hline$p$-Cymene (42) & $\begin{array}{l}\text { L. heterophylla (Essential oil) } \\
\text { L. geoffrayi (Essential oil) }\end{array}$ & $\begin{array}{l}{[43]} \\
{[44]}\end{array}$ \\
\hline$m$-Cymene (43) & L. aromatica (Essential oil) & {$[45,46]$} \\
\hline$\alpha$-Phellandrene (44) & L. conferta (Essential oil) & {$[33]$} \\
\hline$\beta$-Phellandrene (45) & $\begin{array}{l}\text { L. conferta (Essential oil) } \\
\text { L. geoffrayi (Essential oil) }\end{array}$ & $\begin{array}{l}{[33]} \\
{[44]}\end{array}$ \\
\hline$\beta$-Ocimene (46) & $\begin{array}{l}\text { L. conferta (Essential oil) } \\
\text { L. geoffrayi (Essential oil) }\end{array}$ & $\begin{array}{l}{[33]} \\
{[44]}\end{array}$ \\
\hline trans- $\beta$-farnesene (47) & L. conferta (Essential oil) & {$[33]$} \\
\hline
\end{tabular}


(Table 2) contd....

\begin{tabular}{|c|c|c|}
\hline $\begin{array}{l}\text { Compounds } \\
\text { (Str. No.) }\end{array}$ & Source & Ref. \\
\hline$(+)-\beta$-Selinene (48) & L. conferta (Essential oil) & [33] \\
\hline Terpinen-4-ol (49) & L. conferta (Essential oil) & [33] \\
\hline Borneol (50) & L. conferta (Essential oil)L. geoffrayi (Essential oil) & $\begin{array}{l}{[33]} \\
{[44]}\end{array}$ \\
\hline Dihydroumbellulone (52) & L. conferta (Essential oil) & [33] \\
\hline$\alpha$-Eudesmol (53) & L. heterophylla (Essential oil) & [43] \\
\hline$\alpha$-Bulnesene (54) & L. rugosa (Essential oil) & [43] \\
\hline$S$-()-Perillaldehyde (58) & L. geoffrayi (Essential oil) & [44] \\
\hline (+)-cis-Limonene oxide (59) & L. geoffrayi (Essential oil) & [44] \\
\hline cis-Ocimene $(\mathbf{6 0})$ & L. geoffrayi (Essential oil) & [44] \\
\hline
\end{tabular}<smiles>C/C=C/c1ccc(OC)cc1/C=C\C(C)=O</smiles>

61

64<smiles>C/C=C\c1ccc(OC)cc1</smiles>

67<smiles>C=CC(=C)CCC=C(C)C</smiles>

68<smiles>O=CC1CC=CCC1</smiles>

69<smiles>C=CC(=C)CCC=C(C)CCC=C(C)C</smiles>

70<smiles>O=C(O)/C=C/c1ccc(O)c(O)c1</smiles><smiles>O=C(/C=C/c1ccc(O)c(O)c1)O[C@H]1C[C@](O)(C(=O)O)C[C@H](O)[C@H]1O</smiles><smiles>Cc1ccc(C(C)C)c(O)c1</smiles>

73<smiles>C=CCc1ccc(O)c(OC)c1</smiles><smiles>C=CCc1ccc(OC)c(OC)c1</smiles><smiles>C=CCc1ccc(OC)cc1</smiles>

$\mathrm{HCOOH} \quad \mathrm{MeCH}_{2} \mathrm{COOH} \mathrm{MeCOOH} \quad \mathrm{MeCH}_{2} \mathrm{CH}_{2} \mathrm{CH}_{2} \mathrm{COOH}$

$\mathrm{CH}_{3} \mathrm{COCH}_{3} \quad \mathrm{Me}\left(\mathrm{CH}_{2}\right)_{29} \mathrm{CH}_{2} \mathrm{OH}$<smiles>CCCCCC(O)CC</smiles>

81

82

83<smiles>COC1=C(OC)[C@@](O)(C(C)=O)[C@@]2(OC(c3ccc(O)cc3)=CC2=O)O1</smiles>

Fig. (3). Structures of miscellaneous compounds from Limnophila.

Do et al. [58] studied the comparative effect of different solvents (methanol, ethanol and acetone in water) in extracts of L. aromatica and evaluated their antioxidant activity, total phenolic content, and total flavonoid content of the freezedried L. aromatica extracts. The extract obtained by $100 \%$ ethanol was found to exhibit the highest total antioxidant activity, reducing power and DPPH radical scavenging activity. The same extract also exhibited the highest phenolic con- tent (40.5 mg gallic acid equivalent/g of defatted L. aromatica) and the highest flavonoid content (31.11 mg quercetin equivalent/g of defatted L. aromatica); thus, L. aromatica can be useful in dietary applications with a potential to reduce oxidative stress [58].

Recently, Brahmachari and his group [26] reported on the evaluation of antimicrobial potential of two bioflavonoids, i.e., 5,7-dihydroxy-6,8,4'-trimethoxyflavone (nevadensin; 7) 
Table 3. Miscellaneous Compounds of Limnophila.

\begin{tabular}{|c|c|c|}
\hline $\begin{array}{l}\text { Compounds } \\
\text { (Str. No.) }\end{array}$ & Source & Ref. \\
\hline p-Methoxybenzoic acid (61) & L. rugosa (Essential oil) & {$[48]$} \\
\hline Anisaldehyde (62) & L. rugosa (Essential oil) & {$[48]$} \\
\hline Anisylacetone (63) & L. rugosa (Essential oil) & {$[43]$} \\
\hline 3-Hexen-2-one (65) & L. aromatica (Essential oil) & {$[45,46]$} \\
\hline trans-Anethole (66) & L. rugosa (Essential oil) & {$[43]$} \\
\hline cis-Anethole (67) & L. rugosa (Essential oil) & {$[43]$} \\
\hline$\beta$-Myrcene (68) & $\begin{array}{l}\text { L. aromatica }(\text { Essential oil) } \\
\text { L. geoffrayi (Essential oil) }\end{array}$ & $\begin{array}{l}{[45,46]} \\
{[44]}\end{array}$ \\
\hline Caffeic acid (71) & L. aromatica (Essential oil) & {$[23]$} \\
\hline Chlorogenic acid (72) & L. aromatica (Essential oil) & {$[23]$} \\
\hline Thymol (73) & Essential oil of $L$. conferta & {$[33]$} \\
\hline Eugenol (74) & L. aromatica (Essential oil) & {$[23]$} \\
\hline Methyleugenol (75) & L. geoffrayi (Essential oil) & {$[44]$} \\
\hline Methylchavicol (76) & L. rugosa (Essential oil) & {$[48]$} \\
\hline Hentriacontanol (82) & L. rugosa (Essential oil) & {$[41]$} \\
\hline 3-Octanol (83) & L. geoffrayi (Essential oil) & {$[44]$} \\
\hline 1-Octen-3-ol (84) & $\begin{array}{l}\text { L. aromatica (Essential oil) } \\
\text { L. geoffrayi (Essential oil) }\end{array}$ & $\begin{array}{l}{[45-47]} \\
{[44]}\end{array}$ \\
\hline 3-Octanone (85) & L. geoffrayi (Essential oil) & {$[44]$} \\
\hline 1-Decanol (86) & L. geoffrayi (Essential oil) & {$[44]$} \\
\hline Limnophila-spiroketone (87) & L. geoffrayi (aerial parts) & [49] \\
\hline
\end{tabular}

and 5,6-dihydroxy-7,8,4'-trimethoxyflavone (9), isolated from $L$. heterophylla and $L$. indica, respectively, against the microbial strains Bacillus subtilis, Staphylococcus aureus, Escherichia coli, Salmonella typhimurium, Alternaria solani, and Candida albicans. Compounds 7 and 9 exhibited moderate but broad antimicrobial activities against both Grampositive and Gram-negative bacteria and also against the fungal pathogens. Compound 7 showed a bactericidal effect against E. coli and S. aureus (MICs of 200 and $250 \mathrm{mg} / \mathrm{ml}$, respectively), while compound 9 was found to effectively kill $B$. subtilis by cell lysis. The detailed experimental results are presented in Table 6. The scanning electron microscopic pictures (Fig. 4) clearly delineate morphological deformities of the microbial cells observed by the effect of compounds 7 and 9 [26].

The same laboratory of Brahmachari [40] also reported on the isolation of a potent antimicrobial pentacyclic triterpenoid constituent, characterized as 3-oxo-olean12(13),18(19)-dien-29 $\alpha$-carboxylic acid (27) on the basis of detailed spectral studies, from the aerial parts and roots of $L$. indica. The antibacterial effect of the compound 27 was evaluated against three Gram-positive (Bacillus subtilis 
Table 4. Antimicrobial activity of $L$. racemosa and $L$. indica [51].

\begin{tabular}{|c|c|c|c|c|c|c|c|c|}
\hline \multirow{2}{*}{ Plant extracts } & \multicolumn{7}{|c|}{ Diameter of the inhibition zone including diameter of well } \\
$(\mathbf{1 0}$ mm) in mm.
\end{tabular}

*1. Bacillus anthracis 4. Bacillus subtilis 7. Staphylococcus albus

2. Bacillus mycoides $\quad$ 5. Pseudomonas sp. 8.Xanthomonas campestris

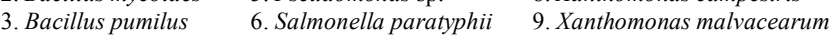

Table 5. Antimicrobial activity of $L$. gratissima [33].

\begin{tabular}{|c|c|c|c|}
\hline Bacteria & \multicolumn{3}{|c|}{ Diameter of inhibition zone (mm) } \\
\hline & $\begin{array}{c}\text { Essential oil } \\
\text { L.gratissima } \\
(\mathbf{0 . 1} \mathbf{m})\end{array}$ & $\begin{array}{c}\text { Chloramphenicol } \\
\text { (positive control) } \\
\mathbf{2 5 \mu}\end{array}$ & $\begin{array}{c}\text { Streptomycin } \\
\text { (positive control) } \\
\mathbf{5 0} \mu \mathbf{g}\end{array}$ \\
\hline \hline Bacillus subtilis & 18 & 18 & 21 \\
\hline Staphylococcus aureus & 16 & 15 & 21 \\
\hline Escherichia coli & 14 & 18 & 23 \\
\hline Pseudomonas aeruginosa & 15 & 17 & 20 \\
\hline
\end{tabular}

Table 6. Anti-microbial study by microdilution method for compounds $7 \&$ 9, and thymol (positive control) against the bacteria $E$. coli, S. typhimurium, $S$. aureus, B. subtilis, and fungi $C$. albicans and $A$. solani, assessed by the Disk Diffusion and the Broth Microdilution Methods. Values are arithmetic means with ranges in parentheses $(n=3)$.

\begin{tabular}{|c|c|c|c|c|c|c|c|c|c|c|c|c|c|}
\hline \multirow{3}{*}{ Microorganism } & \multirow{3}{*}{$\begin{array}{l}\text { Incubation } \\
\text { period (h) }\end{array}$} & \multicolumn{6}{|c|}{ Inhibition zone diameter $(\mathbf{m m})^{\text {a) }}$} & \multicolumn{6}{|c|}{ Minimum inhibitory concentration (MIC) values $(\mu \mathrm{g} / \mathrm{ml})$} \\
\hline & & \multicolumn{2}{|c|}{7} & \multicolumn{2}{|c|}{9} & \multicolumn{2}{|c|}{ Thymol } & \multicolumn{2}{|c|}{7} & \multicolumn{2}{|c|}{9} & \multicolumn{2}{|c|}{ Thymol } \\
\hline & & $\begin{array}{c}\text { Arithmetic } \\
\text { mean }\end{array}$ & Range & $\begin{array}{c}\text { Arithmetic } \\
\text { mean }\end{array}$ & Range & $\begin{array}{c}\text { Arithmetic } \\
\text { mean }\end{array}$ & Range & $\begin{array}{c}\text { Arithmetic } \\
\text { mean }\end{array}$ & Range & $\begin{array}{c}\text { Arithmetic } \\
\text { mean }\end{array}$ & Range & $\begin{array}{c}\text { Arithmetic } \\
\text { mean }\end{array}$ & Range \\
\hline E. coli & 24 & 20 & $15-25$ & $-^{\text {b) }}$ & - & 21 & $19-23$ & 200 & $150-250$ & - & - & 200 & $150-200$ \\
\hline S. typhimurium & 24 & 14 & $12-17$ & 12 & $9-14$ & 18 & $14-21$ & 300 & $250-350$ & 500 & $450-550$ & 225 & $200-260$ \\
\hline S. aureus & 24 & 18 & $16-20$ & - & - & 16 & $14-18$ & 250 & $200-375$ & - & - & 300 & $275-325$ \\
\hline B. subtilis & 24 & - & - & 18 & $15-21$ & 19 & $16-21$ & - & - & 300 & $200-350$ & 350 & $300-400$ \\
\hline C. albicans & 24 & - & - & 19 & $17-21$ & 20 & $17-22$ & - & - & 225 & $200-300$ & 400 & $350-450$ \\
\hline A. solani & 24 & 19 & $18-21$ & - & - & 20 & $18-21$ & 200 & $150-300$ & - & - & 350 & $300-400$ \\
\hline
\end{tabular}

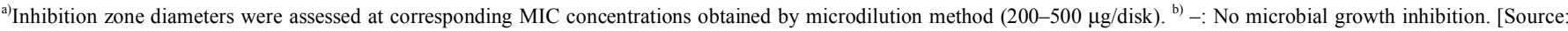
Brahmachari et al., Chem. Biodiv., 2011, 8, 1039-1151; reproduced with permission]

MTCC121, Staphylococcus aureus MTCC96, Listeria monocytogenes MTCC657), Lactococcus lactis subsp. lactis LABW4 and four Gram-negative (Salmonella typhimurium MTCC98, Escherichia coli MTCC1667, Pseudomonas aeruginosa MTCC741, Pantoea ananatis MTCC2307) bacteria. The compound 27 showed its activity against Grampositive bacteria at very low concentrations within a range of
25-30 $\mu \mathrm{g} / \mathrm{ml}$, whereas it showed a variable sensitivity against Gram-negative bacteria. It could kill E. coli and P. aeruginosa at lower concentrations (40 and $30 \mu \mathrm{g} / \mathrm{ml}$, respectively), but a comparatively higher amounts $(75$ and $100 \mu \mathrm{g} / \mathrm{ml}$, respectively) of the compound were required to kill $P$. ananatis and $S$. typhimurium. The overall results are shown in Table 7. Interestingly, the triterpenoid $\mathbf{2 7}$ was found not to 
Fig. 4a)
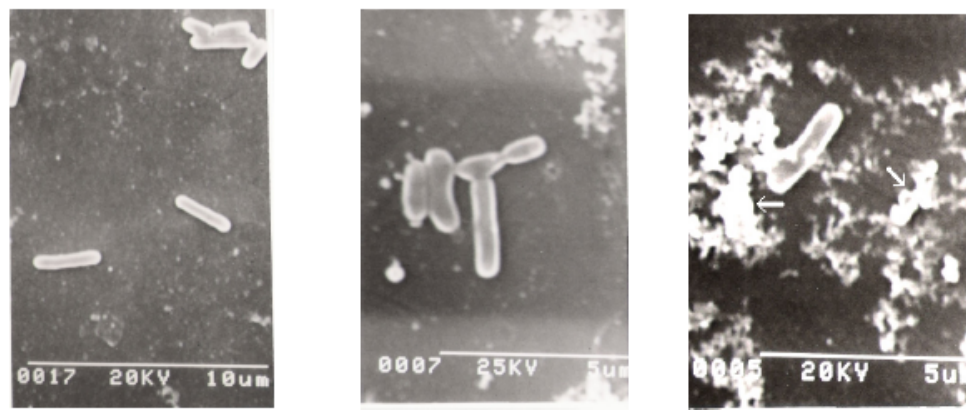

Fig. 4b)
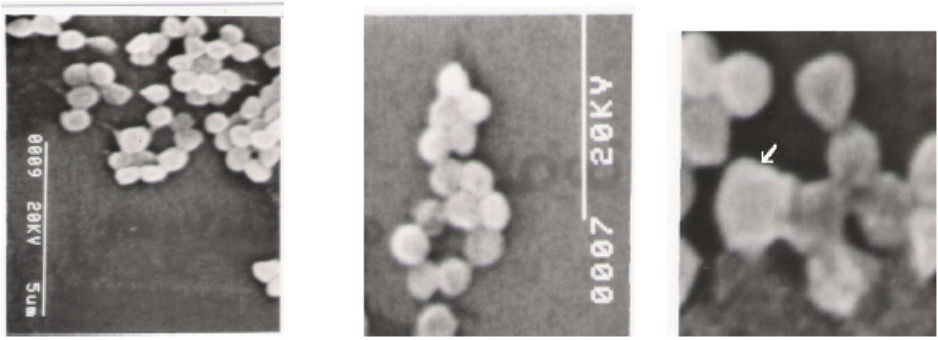

Fig. 4c)
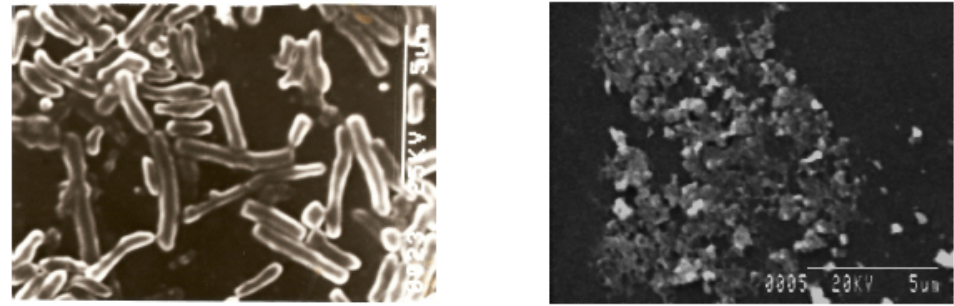

Fig. (4). Scanning electron microscopic pictures: a) effect of compound 7 on the morphology of Escherichia coli, b) effect of compound 7 on the morphology Staphylococcus aureus, and c) effect of compound 9 on the morphology of Bacillus substilis. Arrows indicate morphological deformities of the microbial cells. [Source: Brahmachari et al., Chem. Biodiv., 2011, 8, 1039-1151; reproduced with permission].

Table 7. Antibacterial activity of compound 27 and the positive control oxytetracyclin against the Bacteria $E$. coli, $S$. typhimurium, $S$. aureus, B. subtilis, $P$. aeruginosa, $P$. ananatis and $L$. monocytogenes assessed by the disk diffusion and the broth microdilution methods. Values are arithmetic means with ranges in parentheses $(n=3)$.

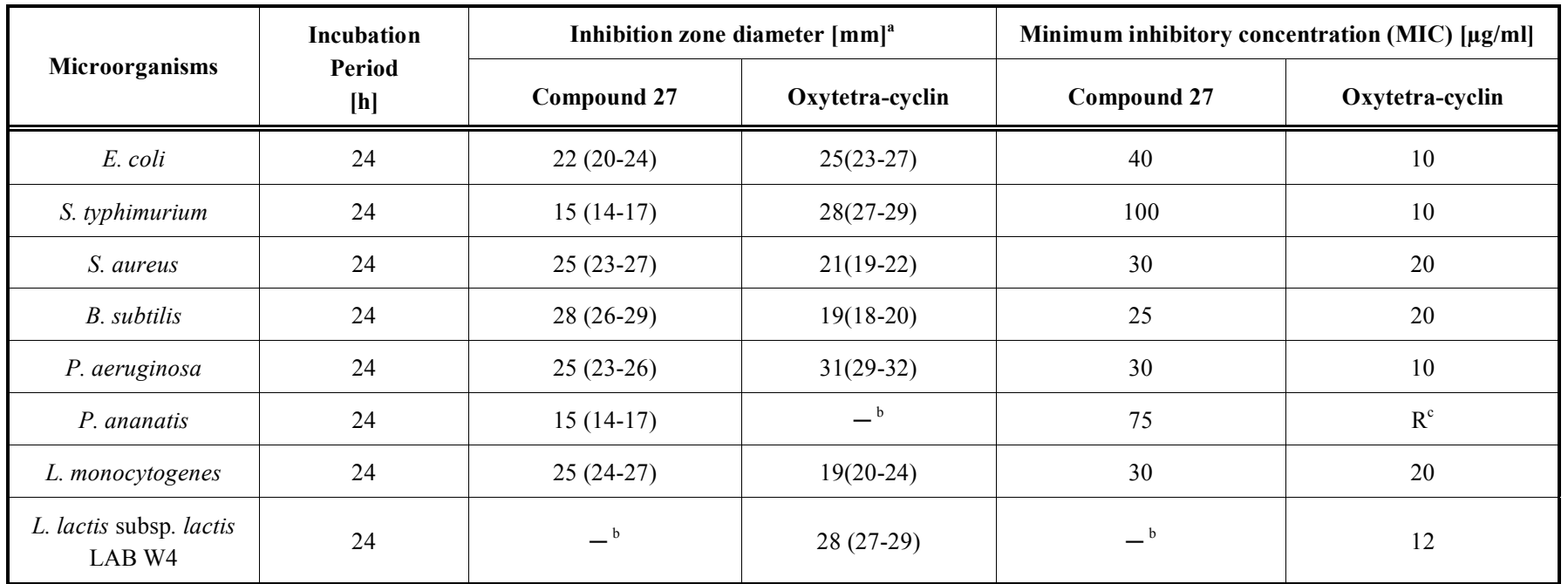

${ }^{\mathrm{a}}$ Inhibition zone diameters were assessed at corresponding MIC concentrations obtained by microdilution method (200-500 mg/disk). ${ }^{\mathrm{b}}-:$ No microbial growth inhibition. ${ }^{\mathrm{c}}$ Resistance. [Source: Brahmachari et al., Fitoterapia, 2013, 90, 104-111; reproduced with permission] 
inhibit a probiotic lactic acid bacterium Lactococcus lactis subsp. lactis LABW4 under in vitro condition and to possess no toxicity in Swiss albino mice [40]. Detailed studies on the mode of action were also reported by this group [40].

\section{Anti-Inflammatory Activity}

Reddy et al. [33] studied the anti-inflammatory activity of the essential oil and crude extract of $L$. conferta and also of nevadensin (a chemical constituent), isolated from the plant, in acute and chronic inflammatory model employing the method of Winter et al. [59]. Carrageenan-induced rat paw edema was compared at ' 0 ' and ' 3 ' hours with that of control (4\% gum acacia mucilage). In tests for acute inflammatory activity, nevadensin (5,7-dihydroxy-6,8,4'trimethoxy- flavone) showed significant inhibition $(\mathrm{P}<$ 0.001 , dose $75 \mathrm{mg} / \mathrm{Kg}$ oral, \% inhibition 45.28) but neither the volatile oil nor the crude extract, showed any significant activity compared to the control. However, in chronic inflammation model, the crude extract of $L$. conferta reduced $(\mathrm{P}<0.001$, dose $500 \mathrm{mg} / \mathrm{Kg} /$ day oral) the weight of dry granuloma (22.1 $\pm 1.4 \mathrm{mg} \%$ of body weight) compared to the control value ( $36 \pm 1.86 \mathrm{mg} \%$ of body weight). Nevadensin (5,7-dihydroxy-6,8,4'-trimethoxyflavone) has recently been reported to have in vitro weak inhibitory activity against cyclooxygenase- 1 and 2 (COX-1 and COX-2) as studied in COX catalyzed prostaglandin biosynthesis assay [60].

\section{Antitubercular Activity}

Nevadensin and isothymusin (6,7-dimethoxy-5,8,4'trihydroxyflavone), isolated from the chloroform extract of the aerial parts of L. geoffrayi, were reported to exhibit growth-inhibitory activity against mycobacterium tuberculosis $\mathrm{H} 37 \mathrm{Ra}$ with equal MIC value of $200 \mu \mathrm{g} / \mathrm{mL}$ [15]; however the efficacy is relatively lower than those of the standard drugs (used during the experiment) rifampicin (MIC 0.003-0.0047 $\mu \mathrm{g} / \mathrm{mL}$ ), isoniazid (MIC 0.025-0.05 $\mu \mathrm{g} / \mathrm{mL}$ ) and kanamycin sulphate (MIC $1.25-2.5 \mu \mathrm{g} / \mathrm{mL}$ ). But the flavone, nevadensin was found to be more effective (MIC values: $100 \mu \mathrm{g} / \mathrm{mL}$ for nevadensin; $10 \mu \mathrm{g} / \mathrm{mL}$ for streptomycin used as standard) against the $\mathrm{H} 37 \mathrm{Rv}$ strain of $M$. tuberculosis as reported by Reddy et al. [33]. The investigators suggested that the compound shows no toxicity up to $600 \mu \mathrm{g} / \mathrm{Kg}$ orally in acute toxicity studies.

\section{Wound Healing Activity}

The crude alcoholic extract of Limnophila conferta was reported to possess wound-healing property [33]. The effect was studied in three different experimental wound models. Animals were wounded under pentobarbitone [40 $\mathrm{mg} / \mathrm{Kg}$ /intraperitoneal(IP)] anesthesia (supplemented with ether) to bear either incision/ or excision/ dead space wound. The crude extract was given in the dose of 500 $\mathrm{mg} / \mathrm{Kg} /$ orally, (once daily) up to 10 days (incision and dead space wound) or until complete healing (excision wound) and the tensile strength was measured on the $10^{\text {th }}$ day.

In the excision wound model, the crude extract showed significant $(\mathrm{P}<0.001)$ reduction on the epithelisation period (17.22 \pm 0.46 days) compared to that of the control (unreacted wounded animals); (21 \pm 0.1 days $)$ and significant inhibition in the rate of wound contraction on the $4^{\text {th }}, 8^{\text {th }}, 10^{\text {th }}$ and $12^{\text {th }}$ days. The $16^{\text {th }}$ day onwards significant enhancement $(\mathrm{P}<0.001)$ in wound contraction $(97.59 \pm 0.64 \%)$ was shown by crude extract compared to that of control (93.2 \pm 1.48$)$. Effects of crude extract on other wound models were insignificant.

\section{Antioxidant Activity}

Suksamrarn et al. [24] reported significant antioxidant activity of chloroform extract of aerial part of Limnophila geoffrayi. Bioassay-guided fractionation of the extract led to the isolation of two pentaoxygenated flavones - one is nevadensin (7) and the other is isothymusin (16), of which only the latter exhibited antioxidant activity against the radical scavenging ability of 1,1-diphenyl-2-picrylhydrazyl (DPPH) with the $\mathrm{IC}_{50}$ value of $7.7 \mu \mathrm{g} / \mathrm{mL}$. The efficacy is almost comparative with the standard antioxidant compound 2,6-di(tert-butyl)-4-methylphenol (BHT, $\left.\mathrm{IC}_{50}=5.7 \mu \mathrm{g} / \mathrm{mL}\right)$.

It is interesting to note that isothymusin (16) while shows strong antioxidant property, nevadensin can't - this contrasting difference in the behaviour may be explained on the basis of structure/activity relationship. The free 4'hydroxy group in isothymusin molecule exerts delocalization with the 4-keto group after the 4'-hydrogen being abstracted. The $p$-hydroquinone nature of the A-ring possibly also contributes to the relatively high antioxidant activity of the compound. It should also be noted that the free 7-hydroxyl group of nevadensin does not exert any radical scavenging activity by similar mechanism to that of the free 4'-hydroxyl group as observed in case of isothymusin; one possible cause may be the steric hindrance developed due to the two adjacent methoxyls, although such effect is not observed in case of BHT. The antioxidant efficacy of isothymusin, isolated from other sources was also established by Wang et al. [61] and also by Kelm et al. [62].

Recently, methanolic extract of L. aromatica and its essential oil as well was found to possess significant in vitro antioxidant properties assessed in DPPH and nitric oxide (NO) radical scavenging, lipid peroxidation, and ferric reducing antioxidant power (FRAP) assays [52,54,63-66]. The results are promising, and are in accordance to the traditional uses of the plant.

\section{Cytotoxic Activity}

The dihydroxytrimethoxyflavone, nevadensin isolated from the plant $L$. conferta was also reported to display moderate cytotoxic activity [33]; the test compound showed $100 \%$ cytotoxicity at a concentration of $75 \mu \mathrm{g} / \mathrm{mL}$ both in Dalton's lymphoma ascites tumour and Ehrlich ascites tumour (using Swiss albino mice model). The compound was found to be more effective than wogonin that showed only $24.1 \%$ cytotoxicity in both the tumours at the same concentration [33]. This findings support the view of Dong et al. that the methoxylated flavones possess moderate cytotoxic activity [66].

\section{Anthelmintic Activity}

From the studies of Reddy group [33] with the essential oil of $L$. conferta on a variety of worms, it appears that the oil might be used as a potent and effective antidote against 
Table 8. Essential oil of $L$. conferta against earth worm model [33].

\begin{tabular}{|c|c|c|c|c|}
\hline Test material/Positive controls & Worms & Dose (mg/mL) & $\begin{array}{c}\text { Time required for } \\
\text { Paralysis (min) }\end{array}$ & $\begin{array}{c}\text { Time required for } \\
\text { death (min) }\end{array}$ \\
\hline \hline Oil of $L$. conferta & Earth & 1.7 & 125 & 142 \\
Piperazine citrate & worm & 4.7 & 188 & 242 \\
Mebendazole & & 180 & 238 \\
\hline
\end{tabular}

Table 9. Essential oil of $L$. conferta against round worm model [33].

\begin{tabular}{|c|c|c|c|}
\hline Test material/Positive controls & Worms & Dose (mg/mL) & $\begin{array}{c}\text { Time required for } \\
\text { death (min) }\end{array}$ \\
\hline \hline Oil of $L$. conferta & & 2.0 & 240 \\
Thymol & Round worm & 2.0 & 378 \\
Mebendazole & & 2.0 & 380 \\
Piperazine citrate & & 2.0 & 323 \\
\hline
\end{tabular}

Table 10. Essential oil of $L$. conferta against tape worm model [33].

\begin{tabular}{|c|c|c|c|}
\hline Test material/Positive control & Worms & Dose (mg/mL) & $\begin{array}{c}\text { Time required for } \\
\text { paralysis \& death (min) }\end{array}$ \\
\hline \hline Oil of $L$. conferta & & 1.7 & 55 \\
Piperazine citrate & tape worm & 1.7 & 165 \\
\hline
\end{tabular}

such parasites. The oil exhibited dose-dependent anthelmintic activity against the test organisms, and in each case the oil was found to be more effective than the standards used. The experimental results are tabulated in Tables (8-10).

\section{CONCLUDING REMARKS}

Limnophila plants are widely distributed world-wide, and find immense applications in traditional systems of medicine in many countries. Although some works on the chemical and pharmacological aspects of these plants have already been done, a major portion remains unexplored. This present resume is an attempt to compile an all round and up-to-date literature covering its botany to ethnobotany, biological and pharmacological studies as well as phytochemicals as reported so far, with a goal to boost the ongoing research in the field of dynamic bioactive natural products directed toward the searches for 'promising leads' in modern drug development processes.

\section{CONFLICT OF INTEREST}

The author confirms that this article content has no conflict of interest.

\section{ACKNOWLEDGEMENTS}

The author is thankful to the UGC, New Delhi (FIST Programme) for financial support.

\section{REFERENCES}

[1] Li, H.L. Scrophulariaceae. In: Flora of Taiwan; Li, H.L.; Liu, T.S.; Huang, T.C.; Koyama, T.; Devol, C.E. Eds.; Epoch Publishing Co., Ltd.: Taiwan 1978; Vol. IV, pp. 551-616.
[2] Matsumura, J.; Hayata, B. A list of Plants. J. Coll. Sci., Imp. Uni. Tokyo, Jpn., 1906, 22, 277.

[3] Philcox, D. A taxonomic revision of the genus Limnophila R. Br. (Scrophulariaceae). Kew Bull., 1970, 24, 101-170.

[4] Yamazaki, T. A revision of the genera Limnophila and Torenia from Indochina. J. Fac. Sci. Univ. Tokyo. III, 1985, 13, 575-624.

[5] Yang, Y.P. A synopsis of aquatic angiospermous plants of Taiwan. Bot. Bull. Acad. Sin., 1987, 28, 191-209.

[6] Wannan, B.S.; Watwerhouse, J.T. A taxonomic revision of the Australian species of Limnophila R. Br. (Scrophulariaceae). Aust. J. Bot., 1985, 33, 367-380.

[7] Yang, Y.-P.; Yen, S.-H. Notes on Limnophila (Scrophulariaceae) of Taiwan. Bot. Bull. Acad. Sin., 1997, 38, 285-295.

[8] http://www.itis.gov/servlet/SingleRpt/SingleRpt?search_topic= TSN\& search_value $=33635($ accessed on 11.09 .2008$)$

[9] http://plants.usda.gov/java/nameSearch?keywordquery=Limnophila \&mode $=$ sciname\&submit. $x=16 \&$ submit. $y=11 \quad$ (accessed on 11.08.2008)

[10] Shi, L.W.S. Limnophila R. Brown, Prodr. 442. 1810. Flora Chin., 1998, 18, 26-28.

[11] Prajapati, N.D.; Purohit, S.S.; Sharma, A.K.; Kumar, T. A Handbook of Medicinal Plants; Agrobios: India, 2003; pp. 316-317.

[12] Ambasta, S.P. The Useful Plants of India, Publications \& Information Directorate, CSIR: New Delhi-110012, 1986.

[13] The Wealth of India (Raw Materials), CSIR: New Delhi, 1962.

[14] Chopra, R.N.; Nayar, S.L; Chopra, I.C. Glossary of Indian Medicinal Plants, CSIR: New Delhi 1956.

[15] Misra, O.P. Botanical identity of Sugandhabala. J. Res. Ind. Med. Yoga Homoeo, 1978, 13, 110-114.

[16] Sinha, R.; Lakra, V. Edible weeds of tribals of Jharkhand, Orissa and West Bengal. Indian J. Tradit Know, 2007, 6, 217-222.

[17] (a) Brahmachari, G.; Jash, S.; Gangopadhyay, A.; Mondal, S. Limnophila rugosa-A new source for Salvigenin. Proc. $42^{\text {nd }}$ Ann. Convention of Chemists, 9-13 ${ }^{\text {th }}$ Feb., Santiniketan, 2006, C10; (b) Sharma, D.; Gupta, V.K.; Brahmachari, G.; Mondal, S.; Gangopadhyay, A. X-ray study of weak interactions in two flavonoids. Bull. Mater. Sci., 2007, 30, 469-475. 
[18] Mukherjee, K.S.; Chakraborty, C.K.; Bhattacharya, D.; Chatterjee, T.P. A new flavone from Limnophila rugosa. Fitoterapia, 1990, LXI, 366-367.

[19] Bui, M.-L.; Grayer, R.J.; Veitch, N.C.; Kite, G.C.; Tran, H.; Nguyen, Q.-C.K. Uncommon 8-oxygenated flavonoids from Limnophila aromatica (Scrophulariaceae). Biochem. Systemat. Ecol., 2004, 32, 943-947.

[20] Mukherjee, K.S.; Chakraborty, C.K.; Chatterjee, T.P. 5-Hydroxy7,8,2',4'-tetramethoxyflavone from Limnophila rugosa. Phytochemistry, 1989, 28, 1778 .

[21] Mukherjee, K.S.; Manna, T.K.; Laha, S.; Brahmachari, G. Chemical investigation of Limnophila heterophylla and Borreria articularis. J. Indian Chem. Soc., 1994, 71, 655-656.

[22] Mukherjee, K.S.; Brahmachari, G.; Manna, T.K.; Mukherjee, P. A methylenedioxy flavone from Limnophila indica. Phytochemistry, 1998, 49, 2533-2534.

[23] Krishnan, S.; Ramachandran Nair, AG. Revised structures of flavonoids from Limnophila gratissimma (Scrophulariaceae). Indian J. Chem., 1999, 38B, 1009-1010.

[24] Suksamrarn, A.; Poomsing, P.; Aroonrerk, N.; Punjanon, T.; Suksamrarn, S.; Kongkun, S. Antimycobacterial and antioxidant flavones from Limnophila geoffrayi. Arch. Pharm. Res., 2003, 26, 816-820.

[25] Brahmachari, G.; Mondal, S.; Jash, S.K.; Mandal, K.S.; Chattopadyay, S.; Gangopadhyay, A. naturally occurring bioactive Oheterocycles: A quest for new sources. Nat. Prod. Indian J., 2006, 2,74-77.

[26] Brahmachari, G.; Mandal, N. C.; Jash, S. K.; Roy, R.; Mandal, L. C.; Mukhopadhyay, A.; Behera, B.; Majhi, S.; Mondal, A.; Gangopadhyay, A. Evaluation of the antimicrobial potential of two flavonoids isolated from limnophila plants. Chem. Biodiv., 2011, 8, 1039-1151.

[27] Liu, M.C.; Chen, Z.S.; Chung, L.C.; Yang, M.S.; Ho, S.T.; Chen, M.T. Studies on hypotensive constituents of Limnophila rugosa. Chung-hua Yao Hsueh Tsa Chih, 1991, 43, 35.

[28] Brahmachari, G.; Gorai, D.; Chatterjee, D.; Mondal, S.; Mistri, B. 5,8-Dihydroxy-6,7,4'-trimethoxyflavone, a novel flavonoid constituent of Limnophila indica. Indian J. Chem., 2004, 43B, 219222.

[29] Mukherjee, K.S.; Gorai, D.; Sohel, S.M.A.; Chatterjee, D.; Mistri, B.; Mukherjee, B.; Brahmachari, G. A new flavonoid from Limnophila rugosa. Fitoterapia, 2003, 74, 188-190.

[30] Brahmachari, G.; Gangopadhyay, A.; Mondal, S.; Gorai, D.; Chatterjee, D. A new flavonoid from Limnophila indica. Proc. $91^{\text {st }}$ Indian Sci. Cong., 3-7 ${ }^{\text {th }}$ Jan., Chandigarh, 2004, Part-III, 44.

[31] Mukherjee, K.S.; Brahmachari, G.; Manna, T.K.; Mukherjee, P. A new flavone from Limnophila heterophylla. J. Indian Chem. Soc., 1998, 75, 260-261.

[32] Srinivasan, K.K.; Srinivasa, A.K. 7-Desmethyl artemetin, a rare flavone from Limnophila gratissima. Fitoterapia, 1998, LIX, 417418

[33] Reddy, G.B.S.; Melkhani,A.B.; Kalyani, G.A.; Rao, J.V.; Shirwaikar, A.; Kotian, M.; Ramani, R.; Aithal, K.S.; Udupa, A.L.; Bhat, G.; Srinivasan, K.K. Chemical and pharmacological investigations of Limnophila conferta and Limnophila heterophylla. Int. J. Pharmacognosy, 1991, 29, 145-153.

[34] Mukherjee, K.S.; Laha, S.; Manna, T.K.; Roy, S.C. Further works on Limnophila rugosa (Roth.) Merill. and Hoppea fasigiata Clarke (Gentianaceae). J. Indian Chem. Soc., 1995, 72, 63-65.

[35] Brahmachari, G.; Sohel, S.M.A.; Gorai, D.; Mondal, S.; Mistri, B. An ethylenedioxy flavonoid carboxylic acid from Limnophila indica. J. Chin. Chem. Soc., 2003, 50, 325-328.

[36] Reddy, N.P.; Reddy, B.A.K.; Gunasekar, D.; Blond, A.; Bodo, B.; Murthy, M.M. Flavonoids from Limnophila indica. Phytochemistry, 2007, 68, 636-639.

[37] Mukherjee, K.S.; Brahmachari, G.; Manna, T.K.; Laha, S. A new triterpene from Limnophila rugosa (Roth.) Merrill. J. Indian Chem. Soc., 1995, 72, 741.

[38] Mukherjee, K.S.; Brahmachari, G.; Manna, T.K. Triterpene from Limnophila heterophylla. Phytochemistry, 1995, 38, 1273-1274.

[39] Mukherjee, K.S.; Brahmachari, G.; Manna, T.K. Chemistry of Flacourtia jangomas, Limnophila heterophylla and Hoppea fastigiata. J. Indian Chem. Soc., 1997, 74, 738-739.
[40] Brahmachari, G.; Mandal, N.C.; Roy, R.; Barman, S.; Ghosh, R.; Sarkar, S.; Jash, S.K.; Mondal, S. A new pentacyclic triterpene with potent antibacterial activity from Limnophila indica Linn. (Druce). Fitoterapia, 2013, 90, 104-111.

[41] Mukherjee, K.S.; Chakraborty, C.K.; Bhattacharya, D.; Chatterjee, T.P.; Bhattaerjee, P. Chemical examination of Limnophila rugosa (Roth.) Merill. and Buddleia neemda B. Ham. J. Indian Chem. Soc., 1990, 67, 89-90.

[42] Mukherjee, K.S.; Laha, S.; Manna, T.K.; Chakraborty, C.K. Chemical investigation on Limnophila rogusa and Pedilanthus tithymaloides. J. Indian Chem. Soc., 1992, 69, 411-412.

[43] Rastogi, R.P.; Mehrotra, B.N. Eds. Compendium of Indian Medicinal Plants, CDRI and NISCOM: New Delhi, 1998, Vol. 4, p. 435.

[44] Thongdon-A, J.; Inprakhon, P. Composition and biological activities of essential oils from Limnophila geoffrayi Bonati. World $J$. Microbiol. Biotechnol., 2009, 25, 1313-1320.

[45] Bhuiyan, M.N.I.; Akter, F.; Chowdhury, J.U.; Begum J. Chemical constituents of essential oils from aerial parts of Adenosma capitatum and Limnophila aromatica. Bangladesh J. Pharmacol., 2010, 5, 13-16.

[46] Chowdhury, J.U.; Bhuiyan, M.N.I.; Begum, J. Constituents of Volatile Oils from Limnophila aromatica and Adenosma capitatum. Bangladesh J. Sci. Ind. Res., 2011, 46, 385-388.

[47] Tucker, A.O.; Maciarello, M.J.; Hendi, M.; Wheeler, K.A. Volatile leaf and stem oil of commercial Limnophila chinensis (Osb.) Merrill ssp aromatica (Lam.) Yamazaki (Scrophulariaceae). J. Essent. Oil Res., 2002, 14, 228-29.

[48] Rastogi, R.P.; Mehrotra, B.N. Eds. Compendium of Indian Medicinal Plants, CDRI and NISCOM: New Delhi, 1998, Vol. 2, p. 415.

[49] Jang, D.S.; Su, B.-N.; Pawlus, A.D.; Jones, W.P.; Kleps, R.A.; Bunyapraphatsara, N.; Fong, H.H.S.; Pezzuto, J.M.; Kinghorn, A.D. Limnophilaspiroketone, a highly oxygenated phenolic derivative from Limnophila geoffrayi. J. Nat. Prod., 2005, 68, 1134-1136.

[50] Dubey, V. Screening of some extracts of medicinal plants for antimicrobial activity. J. Mycol. Plant Pathol., 2002, 32, 266-267.

[51] Mishra, V.; Kandya, A.K.; Mishra, G.P. Screening of some medicinal plants for Antimicrobial activity. Bull. Bot. Soc. Univ. Saugar, 1980, 27, 57-59.

[52] Nanasombat, S.; Teckchuen, N. Antimicrobial, antioxidant and anticancer activities of Thai local vegetables. J. Med. Plants Res., 2009, 3, 443-449.

[53] Panomket, P.; Wanram, S.; Srivorasmas, T.; Pongprom, N. Bioactivity of plant extracts against Burkholderia pseudomallei. Asian Biomed., 2012, 6, 619-623.

[54] Rattanasena, P. Antioxidant and antibacterial activities of vegetables and fruits commonly consumed in Thailand. Pak. J. Biol. Sci., 2012, 15, 877-882.

[55] Kapil, V.B.; Sinha, A.K.; Sinha, G.K. Antibacterial and antifungal study of some essential oils and their constituents from the plants of Kumaon and its Tarai tract. Bull. Med. Ethnobot. Res., 1983, IV, 124-129.

[56] Rao, J.V.; Aithal, K.S.; Srinavasan, K.K. Antimicrobial activity of the essential oil of limnophila gratissima. Fitoterapia, 1989, 60, 376-377.

[57] Subhadra, S.; Gade, G.; Ravindran, V. K.; Emani, V. S.; Parre, S.; Banji, D. In vitro and in vivo evaluation of the inhibitory effect of Limnophila indica (Linn.) Druce on Shigellosis. J. Chin. Integr. Med., 2012, 10, 538-545.

[58] Do, Q. D.; Angkawijaya, A. E.; Tran-Nguyen, P. L.; Huynh , L. H.; Soetaredjo, F. E.; Ismadji, S.; Ju, Y.-H. Effect of extraction solvent on total phenol content, total flavonoids content, and antioxidant activity of Limnophila aromatica. J. Food Drug Anal., 2014, 22, 296-302.

[59] Winter, C.A.; Risley, E.A.; Nuss, G.W. Carrageenin-induced edema in hind paw of the rat as an assay for antiiflammatory drugs. Proc. Soc. Exp. Med., 1962, 111, 544-547.

[60] Brahmachari, G.; Jash, S.K.; Mandal, L.C.; Mondal, A.; Roy, R. Cyclooxygenase (COX) - inhibitory flavonoid from Limnophila heterophylla. Rasayan J. Chem., 2008,1, 288-291.

[61] Wang, H.; Nair, M.G.; Strasburg, G.M.; Booren, A.M.; Gray, J.I. Antioxidant polyphenols from tart cherries (Prunus cerasus). J. Agric. Food Chem., 1999, 47, 840-844. 
[62] Kelm, M.A.; Nair, M.G.; Strasburg, G.M; Dewitt, D.L. Antioxidant and cyclooxygenase inhibitory phenolic compounds from Ocimum sanctum Linn. Phytomedicine, 2000, 7, 7-13.

[63] Sribusarakum, A.; Bunyapraphatsara, N.; Vajragupta, O.; Watanabe, H. Antioxidant activity of Limnophila aromatica Merr. Thai J. Phytopharm., 2004, 11, 11-17.

[64] Kukongviriyapan, U.; Luangaram, S.; Leekhaosoong, K.; Kukongviriyapan, V.; Preeprame, S. Antioxidant and vascular protec- tive activities of Cratoxylum formosum, Syzygium gratum and Limnophila aromatica. Biol. Pharm. Bull., 2007, 30, 661-666.

[65] McDonald, S.; Prenzler, P.D.; Antolovich, M.; Robards, K. Phenolic content and antioxidant activity of olive extracts. Food Chem., 2001,73, 73-84.

[66] Dong, X.P.; Pche, C.T.; Farnsworth, N.R. Cytotoxic Flavonols from Gutierrezia microcephala.J. Nat. Prod., 1987, 50, 337-338.

Received: October 30, 2014

Revised: December 07, 2014

Accepted: December 08, 2014

(C) Goutam Brahmachari; Licensee Bentham Open.

This is an open access article licensed under the terms of the Creative Commons Attribution Non-Commercial License (http://creativecommons.org/licenses/by-nc/3.0/) which permits unrestricted, non-commercial use, distribution and reproduction in any medium, provided the work is properly cited. 